\title{
Performance Analysis of Medical Video Streaming over 4G and Beyond Small Cells for Indoor and Moving Vehicle (Ambulance) Scenarios
}

\author{
Ikram U. Rehman, Student Member, IEEE, Nada Y. Philip, Member IEEE, \\ and Robert S. H. Istepanian, Senior Member IEEE
}

\begin{abstract}
It is believed that small cells will play a very important role in future network (e.g. 5G) to meet the high user requirements in traffic volume, frequency efficiency, and energy and cost reduction. The small cell network is a new paradigm for successful deployment of m-health applications. It can enhance the medical Quality of Service (m-QoS) for the indoor and outdoor end users (e.g. patients and healthcare professionals). This paper investigates the impact of deploying 4G and beyond small cell heterogeneous networks for medical video streaming as an example of $\mathrm{m}$-health application. Furthermore, two different scenarios namely, indoor and vehicular (ambulance) are presented along with their system models and technical requirements. The results show that over all network performance is improved in terms of total femtocells throughput, packet loss and delay of the users who are in the vicinity of the femtocells.
\end{abstract}

Keywords-m-health; 5G; LTE; small cells; Quality of Service;

\section{INTRODUCTION}

Mobile healthcare (m-health) is an evolving paradigm that brings together the evolution of emerging wireless communications and network technologies with the concept of "connected healthcare" [1]. Since the introduction of mobile broadband networks numerous m-health scenarios have been successfully deployed worldwide satisfying the vision of pervasive connected healthcare anytime anywhere [2]. It is well known that the evolution of $4^{\text {th }}$ Generation (4G) mobile network systems will contribute significantly to future mobile healthcare (m-health) applications that require high data rates and more critically reliable diagnostic quality [3]. However, there is an increase in the internet traffic in terms of high bandwidth demanding applications e.g. interactive video streaming, serious gaming and social networking and in particular with the increasing popularity of smart phone devices. According to the recent report by CISCO, internet video is now 40 percent of customer internet traffic, and will reach 62 percent by the end of 2016 [6]. In a shared environment, medical applications will coexists with other applications and often they will use the same wireless technology. This fact leads to some challenges encountered even by $4 \mathrm{G}$ networks, such as the spectral efficiency, energy consumption, high mobility, seamless coverage, varied Quality of service (QoS) and Quality of Experience (QoE) requirements, to name a few [4]. Moreover, $4 \mathrm{G}$ networks have just managed to reach its theoretical data rate limit with existing technologies and therefore are not sufficient enough to overcome the above mentioned issues. In this context, ground breaking wireless technologies are needed to overcome the limitations caused by millions of mobile devices, and for that matter research has already been carried out to explore beyond $4 \mathrm{G}$ or $5 \mathrm{G}$ wireless techniques [4] [5]. Compared to $4 \mathrm{G}$ networks, 5G network should be able to achieve 1000 times the system capacity, 10 times the spectral efficiency, energy efficiency and data rate (i.e. peak data rate of $10 \mathrm{Gbps}$ and $5 \mathrm{Gbps}$ in Downlink (DL) and Uplink (UL) respectively [5]. The overall aim is to provide seamless and ubiquitous communication between people to people, people to machine, machine to machine, regardless of the type of electronic devices/services/networks anytime and anywhere. This means that $5 \mathrm{G}$ networks will revolutionize $\mathrm{m}$-health systems by supporting scenarios not supported by $4 \mathrm{G}$ networks (e.g. transmission of $\mathrm{m}$ health data/image/video from high speed vehicle). In reference to $\mathrm{m}$-health, real-time medical ultrasound video streaming is one such application that will benefit from beyond $4 \mathrm{G}$ or $5 \mathrm{G}$ technology. Furthermore, achieving end-to-end Quality of Service (QoS) from the healthcare perspective and their required levels to guarantee robust and clinically acceptable healthcare services is a challenging issue. To address this issue, 5G cellular architecture separates indoors and outdoors scenarios. The change in the design of cellular architecture is due to the fact that 80 percent of the time mobile users stay indoors, while only 20 percent of time stays outdoors [5]. The current cellular architecture uses an outdoor base station to which mobile users connect, no matter whether they stay indoors or outdoors. This results in high penetration loss for indoor users, as the signals have to go through the building walls to communicate with the outdoor base station, which in turn, reduces data rates, spectral efficiency, and energy efficiency of wireless transmissions. In other words, the overall Quality of Service (QoS) 
is degraded. The 5G cellular architecture should also be heterogeneous that involves a diverse set of short-range and lowerpower base stations, such as microcells, picocells, relays and femtocells, all distributed inside a macrocell [8] The advantage of this new network topology is that the operators no longer need to deploy additional expensive macro base stations usually hard to install in dense urban areas [7].

In this paper, we consider a Heterogeneous Network including a macrocell with a centric eNodeB coexisting with femtocells. Moreover, the main research focus of this paper is to investigate Quality of Service (QoS) analysis of medical video streaming using femtocells as an example of small cell network. Since 5G is a brand-new technology that lacks a reliable simulation platform therefore, the simulation of femtocells is carried out using $4 \mathrm{G}$ LTE-Sim system level simulator. To the best of our knowledge, this topic has not been investigated in literature and will bring novelty to our work.

\section{FEMTOCELLS}

The wireless networks have always been in need of in-expensive solutions to boost indoor coverage. Femtocellular network is one of such solutions [8]. Femtocells are inexpensive home Base stations (BSs) which can be defined as small, low power and higher speed access points that can be installed freely by end users in a plug and play manner at residential, enterprise or hospital premises to get better indoor coverage and improve the throughput of users with reducing the cost of implementation [4][8]. In general, femtocells operate in the licensed spectrum, and have a coverage area over hundred meters. Moreover, each femtocell can accommodate up to 10 users in an urban environment [9]. Femto Base Stations establish connection with standard user equipment (e.g. cell phone, tablets and laptops) over radio interfaces. The user traffic is next sent to the operator's core network through legacy broadband connection which can be an optical fiber or digital subscriber line. In this regard, they relate to $\mathrm{Wi}-\mathrm{Fi}$, but rather they use commercial cellular standards and operate in licensed spectrum. The User Equipment (UE) recognizes femto base station as a regular macro base station [7]. Since the distance between femto base station and its serving users is small therefore, the transmission power of user equipment is saved, resulting in prolonged battery life [4]. The major benefits of femtocellular network over traditional macrocellular network are the improved coverage, reduced infrastructure, reduced power consumptions, improved signal-to-interference noise (SINR) and improved throughput [10]. Femtocells have become a great deal of interest for researchers, which is evident from the increase in number of publications within this field [7] [11]-[22].

\section{MOBILE FEMTOCELLS}

Mobile femtocell (Mfemto) is a new concept of small cell network technology. It is an integration of mobile relays and femtocell [14]. The distinguishing feature of mobile femtocell is that, it can move around and dynamically connect to operator's core network in its vicinity. It utilizes standard radio interface technique to connect with the serving macrocellular base station (eNodeB). To macro base station, Mfemtocell and its connected users act as a single unit. Mfemtocell can be installed in vehicles such as buses, trains and even ambulances. Existing studies [14] show that Mfemtocell can potentially benefit cellular networks by improving spectral efficiency of the network and reducing signaling overhead for various network operations. Most importantly, it can perform handover operations for all its connected users, resulting in reduced handover activities for femtocell users. At present, the users within a vehicle encounter several issues ranging from low SINR, lower throughput, poor signal quality, and multiple handovers to drop connection due to high mobility of vehicle. Mfemtocell can solve these issues [15]. This can be done by making moving vehicle a mobile femtocell, where a user equipment (UE) is connected to inside femto access point as opposed to outside macrocellular network via backhaul wireless channel. Thus, the signal avoids penetration loss from metallic wall of the vehicle, resulting in better quality signal.

A limited number of contributions have been proposed in literature deploying mobile femtocells. To enhance the service quality, the authors in [10] proposed deploying femtocells in vehicles to offer better coverage due to short distance between FBS and User Equipment (UE). The authors in [16] present architecture to provide internet coverage in trains by installing femtocells. The authors in [14] investigate benefits of using mobile femtocells in vehicles. While, the researchers in [10] discussed the same concept and proposed different scenarios for its deployment.

\section{USE CASES}

Envisioning femtocell usage, We first present a set of use cases, using medical video streaming as an example of m-health application, to show the need of femtocells to improve Quality of Service (QoS) for Indoor (Remote site) and Vehicular (Ambulance) environments. Each use case tells a story describing key benefits of femtocell deployment.

\section{A. Indoor Scenario}

The concept of prehospital telecare has been present for almost two decades [17], and has become an integral part of m-health systems [18]. M-health system utilizes the information and communication systems for transmission of $m$-health services from remote indoor site to specialized medical facility at hospital. To that end, m-health system is contributing in improving overall patient's wellbeing by specifically:

- Enabling real-time medical consultations (e.g. teleultrasonogrpahy) for robust diagnostic precision and decisionmaking, for critical medical procedures, and

- Improving preparation at hospital by transferring patient's realtime health information before reaching the hospital.

According to a statistical survey in [20], more than two third of critical incidents occur at indoor sites. This leads to the necessity of reliable broadband wireless technologies for paramedics' staff to seamlessly access m-health applications from remote indoor sites. It is important to note, that end-to-end Quality of Service 
(QoS) has to be maintained throughout the session. Since any degradation in service quality can translate into worsen Quality of Experience (QoE) for $\mathrm{m}$-health experts. For example, the throughput degradation in the wireless link may force high video compression techniques (reduced resolution and/or frame rate) on medical video stream that may affect the credibility of received video [21]. The possible advantages experienced by healthcare professional by accessing femtocellular in indoor are listed as:

- Ability to connect medical UE in both macrocell and femto cell environment.

- Improved coverage in indoor environment.

- A significant increase in throughput per user compared to the macrocell due to lower number of users per femtocell.

- Prioritizing emergency traffic, robust security.

- Ability to move between femtocell and macrocell networks enabling seamless connectivity for $\mathrm{m}$-health services.

\section{B. Ambulance Scenario}

This scenario is extremely important, as the time in between transportation of patient to the medical facility is highly critical, especially when the patient is remote or ambulance is caught in a road traffic [22]. Considerable work has been done in m-health domain for moving vehicle scenarios [22]-[24]. Nevertheless, as m-health applications especially tele-ultrasonography need to overcome several problems due to high mobility of the ambulance, the previous wireless communication technologies limited the medical Quality of Service (m-QoS), making ubiquitous m-health deployment for moving vehicles difficult, since high mobility of vehicle reduces data rates. During the critical time in between transportation of patient to the hospital, the paramedics in an ambulance have to transmit medical data, which may include high resolution images and/or video. Heterogeneous network i.e. mix of macrocell together with femtocells can support wireless mhealth applications in a moving vehicle (even up to $500 \mathrm{~km} / \mathrm{h}$ ) and achieve high uplink data rates, hence supports transmission of high resolution video transmission (tele-ultrasonography) from ambulance to the base station.

\section{SYSTEM MODEL FOR INDOOR SCENARIO}

In this scenario, paramedics are able to continuously take advantage of femtocellular base station resources in the patient's or neighboring location for tele-ultrasonography purposes. In our scenario, it is assumed that the paramedic is using portable $\mathrm{PC} /$ tablet which is interfaced via USB or Bluetooth to peripheral device i.e. portable ultrasound machine. The ultrasound device produces a medical video stream that is to be transmitted to the medical expert located at a hospital. Furthermore, the PC/tablet utilizes radio interface technology to ensure efficient and reliable transmission of medical video stream over $4 \mathrm{G}$ and beyond mobile networks. The indoor layout is assumed for high rise apartments for creating realistic scenario. In the layout the indoor User Equipment (UE) is randomly placed inside the building. The femtocellular base station (HeNB) is assumed to be installed inside one of the rooms in the building. Lastly, the acquired medical ultrasound video stream is then transmitted to operator's core network through broadband connection to medical experts. Figure 1 illustrates end-to-end medical ultrasound streaming scenario using femtocells for Indoor environment.

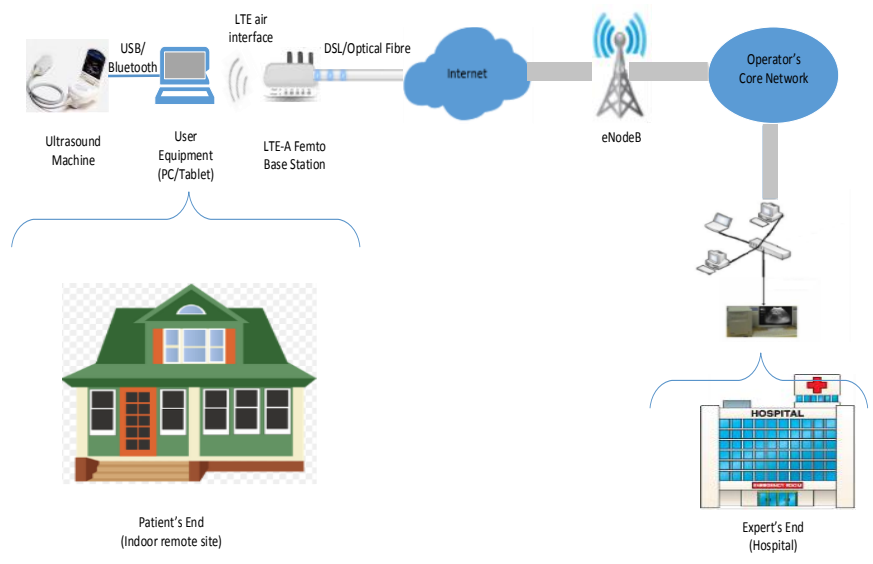

Figure 1: Indoor medical ultrasound streaming scenario.

\section{SYSTEM MODEL FOR AMBULANCE SCENARIO}

In this scenario, we have shown the system model to deploy the femtocellular network inside an ambulance. A Femtocellular base station (FBS) is located inside the ambulance. A transceiver is installed on the roof of the ambulance to transmit/receive data to/from the backhaul macrocellular network. The FBS installed inside the ambulance makes wireless connection between the paramedics and the Femto Access Point (FAP). The FAP and the transceiver are connected through the wired network. The pictorial representation of end-to-end medical ultrasound video streaming for Ambulance scenario is depicted in figure 2.

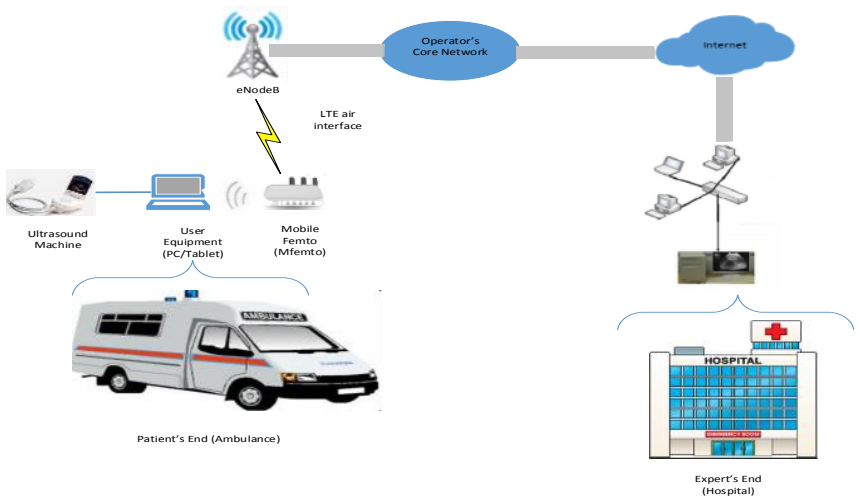

Figure 2: Moving vehicle (Ambulance) scenario for medical ultrasound video streaming. 


\section{Simulation}

In this section, we provide performance analysis of small cell (femto) network communication between femto base station (HeNB) and user. We use a single cell with several femtocells randomly distributed. It is important to note that Mfemto will be considered as fixed femto for the users inside the vehicle, creating an indoor environment, as they will be moving at the same speed as Mfemto. For the video application, we used ultrasound video acquired from Cardiff university hospital. The ultrasound video sequence (at 25 frame/second, resolution 640x480, and YUV format) has been compressed using H.264 standard at the average coding rate of $128 \mathrm{Kbps}$. LTE-Sim simulator is used to perform this analysis [25]. The Quality of Service (QoS) analysis for medical ultrasound video streaming has been carried out with reference to the throughput, packet loss rate and delay. Two different cases are object of this study:

1. Traditional Urban environment without femtocells, where only one macrocell is used as reference case.

2. Urban environment with femtocells.

Table Uplink simulation parameters

\begin{tabular}{|cc|}
\hline Parameters & Values \\
\hline Simulation duration & $100 \mathrm{~s}$ \\
\hline Frame structure & FDD \\
\hline Bandwidth & $15 \mathrm{MHz}$ \\
\hline Macro BS TX power & $43 \mathrm{dBm}$ \\
\hline Femto BS TX power & $20 \mathrm{dBm}$ \\
\hline Maximum delay & $100 \mathrm{~ms}$ \\
\hline $\begin{array}{c}\text { Video bitrate } \\
\text { femtocell }\end{array}$ & $128 \mathrm{Kbps}$ \\
\hline \begin{tabular}{c} 
Scheduling algorithm \\
\hline
\end{tabular} & FME 8 \\
\hline
\end{tabular}

\section{RESULTS AND ANALYSIS}

In this section we compare the performance of traditional macrocellular network and proposed femtocellular network for transmission of medical video streaming in heterogeneous environment under typical traffic and load conditions. The performance is evaluated using key performance indicators (KPIs) i.e. throughput, packet loss rate and delay.

\section{A. Packet loss ratio}

In terms of QoS, PLR is an important parameter of real time flows. PLR increases when scheduler is unable to transmit the real time packets in timely manner. It is evident from figure 3 that PLR increases with the increase in number of users due to higher network load. The accepted PLR for video flows is less than $1 \%$. Unfortunately the PLR shown in figure is higher than $11 \%$ when medical UE is trying to transmit medical video in presence of 80 users under traditional macrocellular environment. This increase in PLR is majorly caused by penetration loss from the walls. On the other hand, femtocellular network performs a PLR way below acceptable value of $1 \%$, in fact almost null.

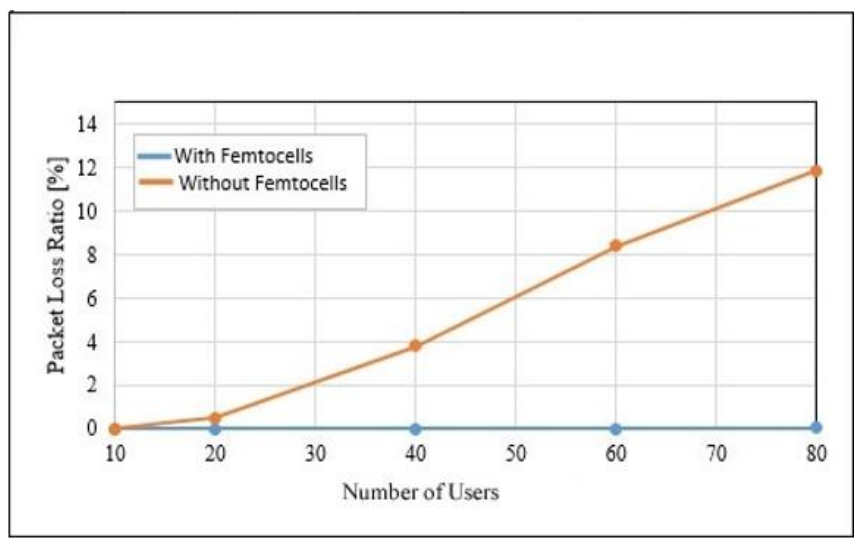

Figure 3. Packet Loss ratio

\section{B. Throughput}

In Figure 4, we compare the throughput curves for both scenarios i.e. with and without the use of femtocells, and we find that the aggregated throughput of users within femtocells linearly increases and reaches to $10 \mathrm{Mbps}$ as opposed to $7.75 \mathrm{Mbps}$ for maximum 80 users considered in our scenario. Figure shows that the overall system capacity can be greatly improved thanks to the adoption of femtocells. Furthermore, figure 5 represents the average throughput for medical video flow. In this figure, we can realize that the transmission of medical video flow in presence of different traffic flows without using femtocells experiences a significant decrease compared to the case when femtocells are active. It is important to note that medical video bitrate in our scenario is set to be $128 \mathrm{Kbps}$

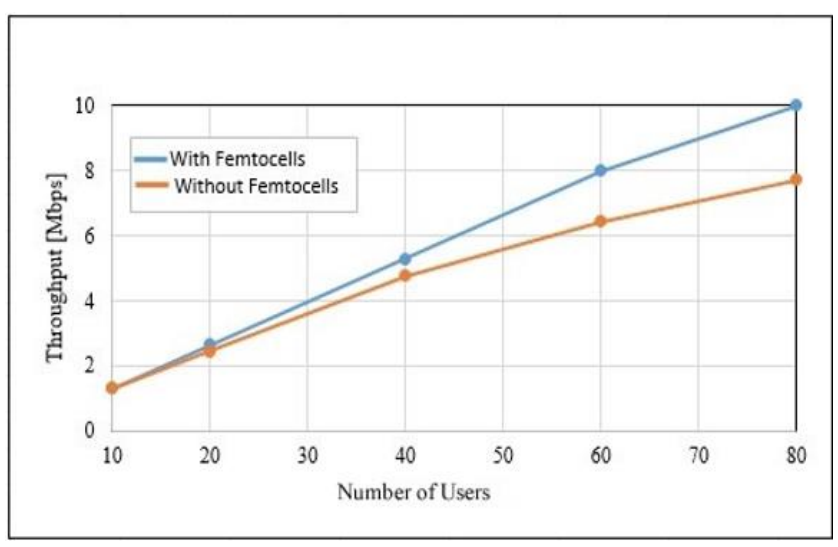

Figure 4. Aggregated Throughput. 


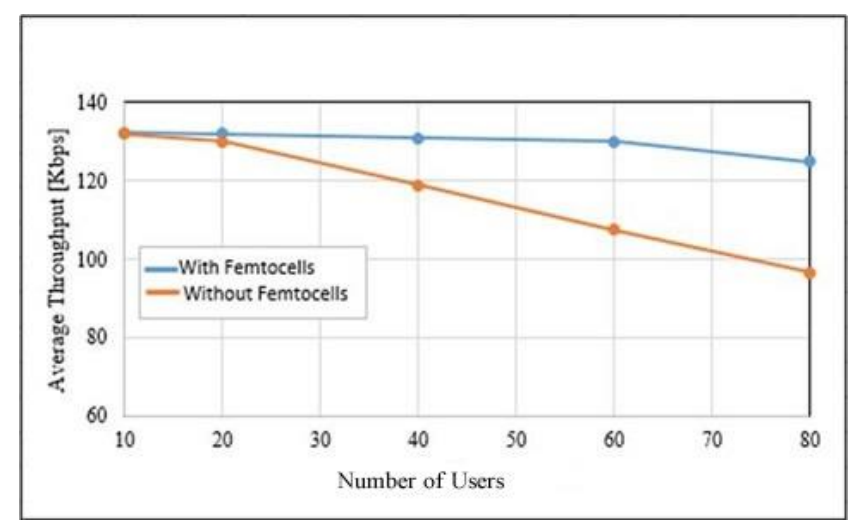

Figure 5. Average Throughput per flow

\section{Delay}

Figure 6 represents delay for video flows. The curve with femtocells show shorter delays than the curve without femtocell. This behavior can be justified by the fact that avoiding penetration losses result in reduced retransmission rate, and hence shorter delays. Moreover, this curve supports the explanation of increase in throughput and decrease in PLR presented in figure 4 and figure 5 respectively.

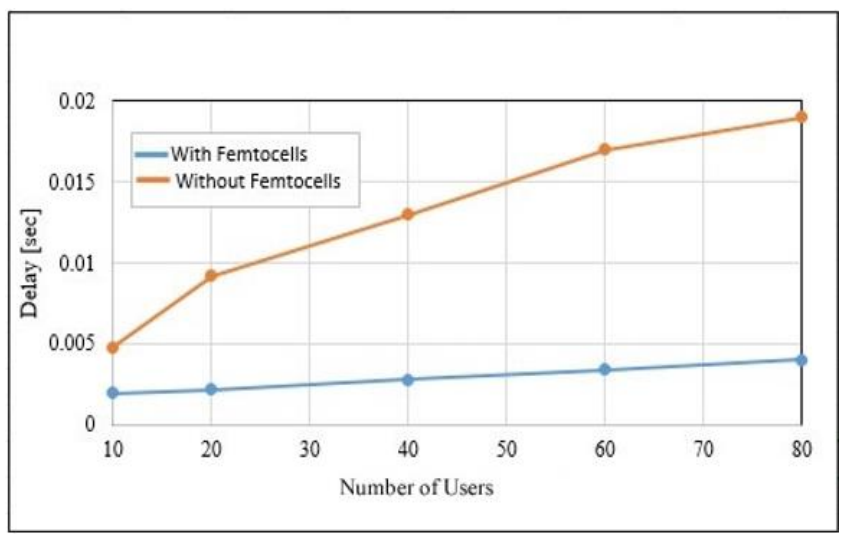

Figure 6. Delay for video flows

\section{CONCLUSION AND FUtURE WORK}

This paper presented a set of use cases and feasibility of deploying small cell heterogeneous networks in the field of mobile health (mhealth) over $4 \mathrm{G}$ and beyond networks. The integration of femtocells as an example of small cell networks with the macro base station will offer high value added services for end users from healthcare perspective. Beyond $\mathrm{m}-\mathrm{QoS}$ enhancement that are the primary target of femtocells, this concept will revolutionize the way wireless connectivity is provided to patients and healthcare professionals. The simulation results indicated that, the use of femtocells in the m-health field offered significant performance advantages in comparison to the conventional macrocellular network case.

Future studies include m-QoS mapping to m-QoE, context awareness. Furthermore, we will revise the study to evaluate the performance benefits of cross layer design, interference mitigating, scheduling, security and self-organizing network techniques in multi cellular heterogeneous environments. The overall objective of our future study will be to present a complete set of small cellular system that surpasses the minimum expected requirements for robust and critical $\mathrm{m}$-health applications.

\section{REFERENCES}

[1] Istepanian, Robert SH, Emil Jovanov, and Y. T. Zhang. "Guest editorial introduction to the special section on m-health: Beyond seamless mobility and global wireless health-care connectivity." Information Technology in Biomedicine, IEEE Transactions on 8, no. 4 (2004): 405-414.

[2] Martini, Maria G. "Wireless broadband multimedia health services: current status and emerging concepts." In Personal, Indoor and Mobile Radio Communications, 2008. PIMRC 2008. IEEE 19th International Symposium on, pp. 1-6. IEEE, 2008.

[3] Istepanaian, Robert SH, and Y-T. Zhang. "Guest editorial introduction to the special section: 4G health-the long-term evolution of m-health." Information Technology in Biomedicine, IEEE Transactions on 16, no. 1 (2012): 1-5.

[4] [4] Cheng Xiang, Fourat Haider, Xigi Gao, Xiao-Hu You, Yang Yang, Dongfeng Yuan, Hadi M. Aggounr, Harald Haas, Simon Fletcher, Erol Hepsaydir "Cellular Architecture and Key Technologies for 5G Wireless

Communication Networks. 5G Wireless Communication Systems: Prospects and Challenges, IEEE Communication Magazine, February 2014.

[5] Shanzhi Chen, Jian Zhao. "The Requirements, Challenges, and technologies for $5 \mathrm{G}$ terrestrial mobile telecommunication", $5 \mathrm{G}$ Wireless Communication Systems: Prospects and Challenges, IEEE Communication Magazine, May 2014.

[6] G. Piro et al. "Simulating lte cellular systems: an open source framework".IEEE Trans. Veh. Tech., vol. 60, pp. 498-513, Oct. 2010.

.[7] Andrews, Jeffrey G., Holger Claussen, Mischa Dohler, Sundeep Rangan, and Mark C. Reed. "Femtocells: Past, present, and future." Selected Areas in Communications, IEEE Journal on 30, no. 3 (2012): 497-508.

[8] Mutafungwa, E., Zhong Zheng, J. Hämäläinen, M. Husso, and T. Korhonen. "Exploiting femtocellular networks for emergency telemedicine applications in indoor environments." In e-Health Networking Applications and Services (Healthcom), 2010 12th IEEE International Conference on, pp. 283-289. IEEE, 2010

[9] Boccuzzi, Joseph, and Michael Ruggiero. Femtocells: design \& application. McGraw Hill Professional, 2010.

[10] Chowdhury, Mostafa Zaman, Seung Que Lee, Byung Han Ru, Namhoon Park, and Yeong Min Jang. "Service quality improvement of mobile users in vehicular environment by mobile femtocell network deployment." In ICT Convergence (ICTC), 2011 International Conference on, pp. 194-198. IEEE, 2011.

[11] Chandrasekhar, Vikram, Jeffrey G. Andrews, and Alan Gatherer. "Femtocell networks: a survey." Communications Magazine, IEEE 46, no. 9 (2008): 59-67.

[12] Joshi, Shaunak, Ray CC Cheung, Pooya Monajemi, and John D. Villasenor. "Traffic-based study of femtocell access policy impacts on HSPA service quality." In Global Telecommunications Conference, 2009. GLOBECOM 2009. IEEE, pp. 1-6. IEEE, 2009. 
[13] Kim, Ronny Yongho, Jin Sam Kwak, and Kamran Etemad. "WiMAX

femtocell: requirements, challenges, and solutions." Communications Magazine, IEEE 47, no. 9 (2009): 84-91.

[14] Haider, Fourat, Mehrdad Dianati, and Rahim Tafazolli. "A simulation based study of Mobile Femtocell assisted LTE networks." In Wireless Communications and Mobile Computing Conference (IWCMC), 2011 7th International, pp. 21982203. IEEE, 2011.

[15] Chowdhury, M. Zaman, Sung Hun Chae, and Yeong Min Jang. "Group handover management in mobile femtocellular network deployment." In Ubiquitous and Future Networks (ICUFN), 2012 Fourth International Conference on, pp. 162-165. IEEE, 2012.

[16] Noriega-Vivas, Patricia, Celeste Campo, Carlos Garcia-Rubio, and Alicia Rodriguez-Carrion. "MOFETA: a network architecture based on MObile FEmtocells to enhance cellular connectivity on trains." In Communication Technologies for Vehicles, pp. 174-185. Springer Berlin Heidelberg, 2012.

[17] The Role of Communication Systems in Emergency Medical Services

[18] Keane, Margaret Geraldine. "A review of the role of telemedicine in the accident and emergency department." Journal of telemedicine and telecare 15, no. 3 (2009): 132-134.

[19] Pattichis, C. S., E. Kyriacou, S. Voskarides, M. S. Pattichis, R. Istepanian, and $\mathrm{Cl}$ N. Schizas. "Wireless telemedicine systems: an overview." Antennas and Propagation Magazine, IEEE 44, no. 2 (2002): 143-153.

[20] Leung, L. P., C. M. Lo, and H. K. Tong. "Prehospital resuscitation of out-ofhospital cardiac arrest in Queen Mary Hospital." Hong Kong J Emerg Med 7, no. 4 (2000): 191-6.

[21] Kim, Dong-Keun, Sun K. Yoo, Ho-Hyun Kang, In-Cheol Park, Yoo S. Youn, and Seung H. Kim. "Evaluation of compressed video-images for emergency telemedicine work with trauma patients." Journal of telemedicine and telecare10, no. suppl 1 (2004): 64-66.

[22] Batistatos, M. C., George V. Tsoulos, and Georgia E. Athanasiadou. "Mobile telemedicine for moving vehicle scenarios: Wireless technology options and challenges." Journal of Network and Computer Applications 35, no. 3 (2012): $1140-1150$.

[23] Pavlopoulos, Sotiris, Efthyvoulos Kyriacou, Alexandros Berler, Spiridon Dembeyiotis, and Dimitrios Koutsouris. "A novel emergency telemedicine system based on wireless communication technology-AMBULANCE."Information Technology in Biomedicine, IEEE Transactions on 2, no. 4 (1998): 261-267.

[24] Sutjiredjeki, Ediana, Soegijardjo Soegijoko, Tati Latifah R. Mengko, and Suhartono Tjondronegoro. "Development of a mobile telemedicine system with multi communication links for urban and rural areas in Indonesia." In 3rd Kuala Lumpur International Conference on Biomedical Engineering 2006, pp. 660-663. Springer Berlin Heidelberg, 2007.

[25] G. Piro et al. "Simulating lte cellular systems: an open source framework".IEEE Trans. Veh. Tech., vol. 60, pp. 498-513, Oct. 2010 